\title{
Benign Middle Ear Neoplasm
}

National Cancer Institute

\section{Source}

National Cancer Institute. Benign Middle Ear Neoplasm. NCI Thesaurus. Code C4602.

A non-metastasizing neoplasm arising from the middle ear. Representative examples

include middle ear polyp and adenoma. 